EPJ Web of Conferences 112, 02013 (2016)

DOI: $10.1051 /$ epjconf/201611202013

(C) Owned by the authors, published by EDP Sciences, 2016

\title{
Linear vs non-linear QCD evolution in the neutrino-nucleon cross section
}

\author{
Javier L. Albacete ${ }^{1, a}$, José I. Illana ${ }^{1, b}$ and Alba Soto-Ontoso ${ }^{1, c}$ \\ ${ }^{1}$ CAFPE and Departamento de Física Teórica y del Cosmos, Universidad de Granada, \\ E-18071 Campus de Fuentenueva, Granada, Spain.
}

\begin{abstract}
Evidence for an extraterrestrial flux of ultra-high-energy neutrinos, in the order of $\mathrm{PeV}$, has opened a new era in Neutrino Astronomy. An essential ingredient for the determination of neutrino fluxes from the number of observed events is the precise knowledge of the neutrino-nucleon cross section. In this work, based on [1], we present a quantitative study of $\sigma_{v N}$ in the neutrino energy range $10^{4}<E_{v}<10^{14} \mathrm{GeV}$ within two transversal QCD approaches: NLO DGLAP evolution using different sets of PDFs and BK small- $x$ evolution with running coupling and kinematical corrections. Further, we translate this theoretical uncertainty into upper bounds for the ultra-high-energy neutrino flux for different experiments.
\end{abstract}

\section{Introduction}

In 2013, the IceCube Collaboration [2] announced the detection of 28 neutrino events of astrophysical origin from $\mathrm{TeV}$ to $\mathrm{PeV}$ energies. To probe this region of UHE a new generation of experiments is already operating or being deployed. Thus, the Auger collaboration has recently reported on the limits of neutrino fluxes from $10^{8}$ to $10^{10} \mathrm{GeV}$ in [3]. Also, the LUNASKA project [4] will perform related measurements at even higher energies $\left(E_{v} \geq 10^{12} \mathrm{GeV}\right)$ using the Square Kilometre Array [5]. Among the possible astrophysical scenarios, cosmogenic neutrinos could be produced in the scattering of protons off cosmic microwave background photons that would result in a non-negligible flux of neutrinos impinging on Earth up to energies of $E_{v} \sim 10^{12} \mathrm{GeV}$. Neutrinos with higher energies may arise in top-down models of UHE cosmic rays.

The inelastic interaction of neutrinos with nucleons is described in terms of charged current (CC) and neutral current (NC) interactions, which proceed through $W^{ \pm}$and $Z^{0}$ exchanges, respectively. The expression of the differential cross section in the fixed-target frame is:

$$
\begin{aligned}
\frac{\mathrm{d}^{2} \sigma_{v N}^{\mathrm{CC}, \mathrm{NC}}}{\mathrm{d} x \mathrm{~d} y} & =\frac{G_{F}^{2} s}{\pi}\left(\frac{M_{i}^{2}}{M_{i}^{2}+Q^{2}}\right)^{2} \\
& \times\left[x y^{2} F_{1}^{\mathrm{CC}, \mathrm{NC}}\left(x, Q^{2}\right)+(1-y) F_{2}^{\mathrm{CC}, \mathrm{NC}}\left(x, Q^{2}\right)+x y\left(1-\frac{y}{2}\right) F_{3}^{\mathrm{CC}, \mathrm{NC}}\left(x, Q^{2}\right)\right]
\end{aligned}
$$

\footnotetext{
ae-mail: albacete@ugr.es

be-mail: jillana@ugr.es

ce-mail: aontoso@ugr.es
} 
where $G_{F}$ is the Fermi coupling constant, $E_{v}$ is the neutrino energy, $s=2 M_{N} E_{v}$ with $M_{N}$ the nucleon mass and $M_{i}$ denotes the mass of the charged or neutral gauge boson exchanged. The information about the partonic structure of the nucleon is encoded in the structure functions $F_{i}\left(x, Q^{2}\right)$. The kinematics of this process is described in terms of the virtuality of the gauge boson $Q^{2}$, the fraction of the nucleon's momentum carried by the struck quark Bjorken- $x$ and the inelasticity $y=Q^{2} /(x s)$. The integral of the differential cross section Eq. 1 is dominated by values of $Q^{2} \sim M_{i}^{2}=10^{4} \mathrm{GeV}^{2}$. Hence the typical $x$ value probed is $x \sim M_{i}^{2} / 2 M_{N} E_{v}$. For $E_{v}>10^{8} \mathrm{GeV}$ this translates into $x<10^{-5}$, that is, we are going to explore the region of small- $x$ and $Q^{2}$ values of $\sim M_{Z, W}^{2}$. This kinematic region hasn't been probed so far in ground based accelerators such as HERA or the LHC. Thus, the study of $\sigma_{v N}$ provides a test for the different QCD frameworks that describe the scale dependence of parton distribution functions. In particular, we have computed $\sigma_{v N}$ within two orthogonal QCD approaches: NLO DGLAP and running coupling BK evolution. Clearly, the reliability of either approach in this intermediate kinematic region cannot be determined on a priori theoretical arguments. This is so because, at a parametric level, one expects that both large logarithmic corrections $\sim \ln Q^{2}$ and $\ln 1 / x$ resumed in either case to be relevant in that kinematic regime. In our view the use of one or another QCD evolution scheme should be considered as a systematic uncertainty associated to the theoretical estimate of the $v N$ cross section.

\section{Improved parton model and DGLAP evolution}

In the QCD improved parton model the structure functions $F_{i}\left(x, Q^{2}\right)$ are linear combinations of parton distribution functions. We have chosen two sets of PDFs, NNPDF3.0 [6] and MSTW08 [7]. Both sets correspond to DGLAP evolution at NLO accuracy.

In our previous discussion about the kinematical region explored in the neutrino-nucleon interaction we have indicated that high $E_{v}$ is equivalent to small values of $x$. The most recent update of the experimental DIS data available [6] explores $x$ down to $\sim 10^{-5}-10^{-6}$. The PDFs are essentially unconstrained below this barrier and these uncertainties are fully propagated to the neutrino-nucleon cross section. This uncertainty, however, is not universal for all the PDFs collaborations even though they use an analogous procedure: an initial parametrization for the PDF that is fitted to data followed by DGLAP evolution. Not only the error bands but also the central value changes from one set to another, as it is displayed in Fig. 1 [1]. In the left plot of Fig. 1 it is shown the neutrino-nucleon cross section calculated with the MSTW08 PDF set. It can be seen how the error bands increase while rising the neutrino energy due to the increasing contribution of small- $x$ values reaching a $30 \%$ uncertainty for the highest energy. The right plot shows the ratio between the cross section obtained with the MSTW08 and NNPDF3.0 PDF sets, both for charged and neutral currents. The prediction with the NNPDF3.0 set is higher for $E_{v} \gtrsim 10^{8} \mathrm{GeV}$ becoming incompatible with the MSTW08 set within the error band at the highest neutrino energy. This illustrates the decreasing predictive power of the approaches based on DGLAP when extended in the $x$-direction to values smaller than those included in their fitting data sets.

\section{Dipole model and BK evolution}

In the dipole model of DIS at low- $x$, the neutrino-nucleon cross section affords the following physics interpretation: long before reaching the target, the exchanged electroweak boson fluctuates into a colourless quark-antiquark dipole. Subsequently, the $q \bar{q}$ dipole scatters off the hadronic target via multiple gluon exchanges. The DIS structure functions for neutrino-nucleon scattering in the dipole 

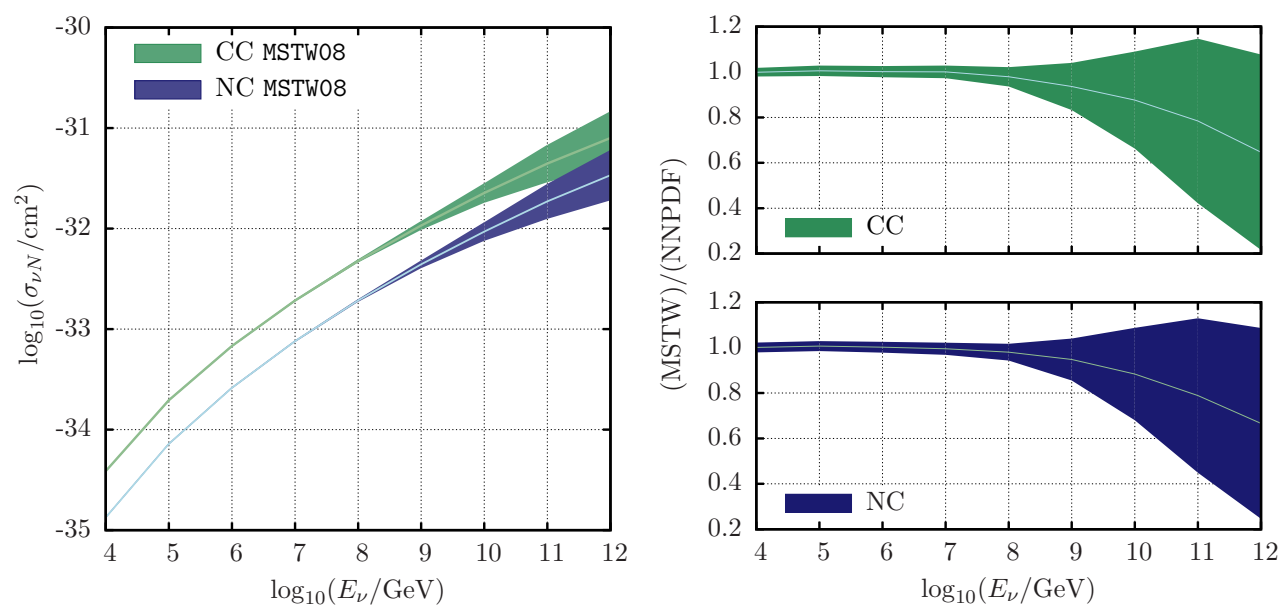

Figure 1. Left: the neutrino-nucleon cross section at NLO with MSTW08 PDFs. Right: comparison between the results for $\sigma_{v N}$ obtained with MSTW08 and NNPDF3.0.

model are given by

$$
F_{\mathrm{T}, \mathrm{L}}^{\mathrm{CC}, \mathrm{NC}}\left(x, Q^{2}\right)=\frac{Q^{2}}{4 \pi} \int \mathrm{d}^{2} \mathbf{b} \mathrm{d}^{2} \mathbf{r} \int_{0}^{1} \mathrm{~d} z\left|\psi_{\mathrm{T}, \mathrm{L}}^{W^{ \pm}, Z^{0}}\left(z, Q^{2}, \mathbf{r}\right)\right|^{2} \mathcal{N}_{\mathrm{dip}}(x, \mathbf{r}, \mathbf{b}) .
$$

All the information about the strong interactions in Eq. (2) is encoded in $\mathcal{N}_{\text {dip }}(x, \mathbf{r}, \mathbf{b})$, the dipolehadron scattering matrix. Although of ultimate non-perturbative origin, the evolution of the dipole scattering amplitude towards smaller values of $x$ can be studied perturbatively via the BK equation. It reads

$$
\frac{\partial \mathcal{N}(r, x)}{\partial \ln \left(x_{0} / x\right)}=\int \mathrm{d}^{2} \mathbf{r}_{1} \mathcal{K}\left(r, r_{1}, r_{2}\right)\left[\mathcal{N}\left(r_{1}, x\right)+\mathcal{N}\left(r_{2}, x\right)-\mathcal{N}(r, x)-\mathcal{N}\left(r_{1}, x\right) \mathcal{N}\left(r_{2}, x\right)\right]
$$

where $x_{0}$ is the initial value of Bjorken- $x$ and $\mathcal{K}$ is the evolution kernel, which plays an analogous role to the DGLAP splitting functions. The evolution kernel is currently known to NLO accuracy in $\alpha_{s} \ln 1 / x$ [8] and also at running coupling accuracy [9-11]. However, NLO BK evolution turns unstable for a large class of initial conditions [12]. Therefore, we have solved the BK equation either at running coupling accuracy and also adding double logarithmic corrections recently calculated in $[13,14]$ to the evolution kernel. We have used the parametrizations of the dipole scattering amplitude obtained in [15, 16] and [17] after fitting all available data on the reduced cross sections measured in $e p$ collisions at HERA with $x<10^{-2}$. The kernel was evaluated at running coupling accuracy (RUN) and including large double transverse logarithmic corrections (DLA). For comparison, we shall also calculate the neutrino-nucleon cross section using the GBW [18], IIM [19] and Soyez [20] phenomenological models for the quark-antiquark dipole cross section (PHENO).

We present in Fig. 2 our results for the $v N$ total cross section as a function of the neutrino energy calculated within the dipole model. We observe that the DLA parametrizations yield a systematically smaller cross section than the RUN ones. The differences between the two are of a factor $\lesssim 2$ for $E_{v}=10^{14} \mathrm{GeV}$. This is an expected result, since the main role of DLA corrections is to further reduce the phase-space for small- $x$ gluon emission, thus resulting in a slower growth of the dipole amplitude with decreasing values of $x$. Finally, the results obtained from the PHENO parametrizations yield an 


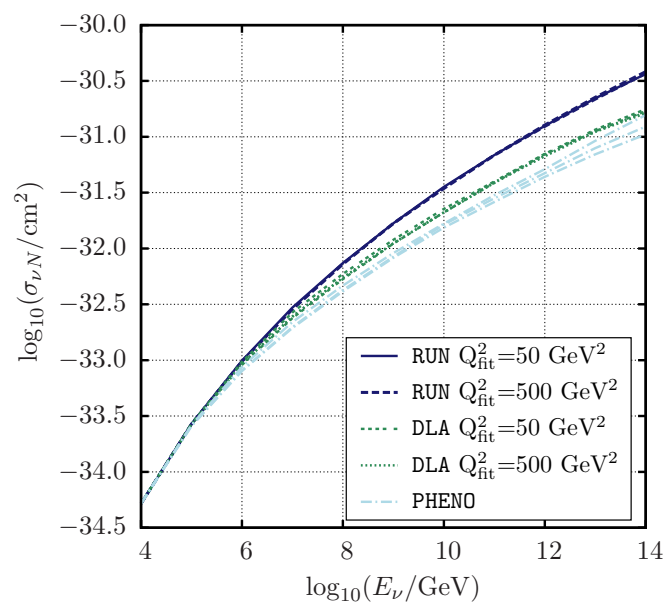

Figure 2. The neutrino-nucleon cross section calculated within the dipole model using different parametrizations for the dipole cross section, $\sigma_{q \bar{q}}$ : RUN, DLA and PHENO.

even smaller value of the cross section at high energies. Once the evolution kernel for BK is fixed, the value of $\sigma_{v N}$ is almost insensitive to the initial conditions for the BK equations. This is seen by comparing the predictions for either DLA or RUN parametrizations fitted to different subsets of HERA data, up to $Q_{\mathrm{fit}}^{2}=50$ and $500 \mathrm{GeV}^{2}$ respectively. We conclude that the main systematic uncertainty in the calculation of $\sigma_{v N}$ within the dipole model stems from the precise dynamical input embodied in the evolution kernel, and not from the initial conditions for the evolution.

\section{DGLAP vs BK}

The main result of our study is shown in Fig. 3. In this plot we compare the values for $\sigma_{v N}$ obtained under the DGLAP and BK QCD evolution schemes. The band for the DGLAP based calculation originates from the uncertainties of the NNPDF3.0 PDF set used here. In turn, the uncertainty band associated to the BK-based results is related to the choice of running coupling or running coupling plus DLA corrections evolution schemes for the high-energy extrapolation. We see that the DGLAP and BK approaches yield similar results for $\sigma_{v N}$ up to energies $E_{v} \sim 10^{8} \mathrm{GeV}$. For higher neutrino energies, the BK-based approaches lead to a systematically smaller value of the cross section than the DGLAP based calculation. That difference between DGLAP and BK approaches become as high as a factor $\sim 4.5$ at the highest neutrino energy $E_{v}=10^{14} \mathrm{GeV}$.

\section{Astrophysical implications}

In absence of a unified theoretical framework for the description of QCD scattering in the full kinematic $\left(x, Q^{2}\right)$-plane, the differences in the theoretical prediction of the total neutrino-nucleon cross section induce an uncertainty in the determination of the upper bounds of astrophysical neutrino fluxes. The event rate at a neutrino telescope is given by

$$
N_{\text {evt }}=\sum_{i=1,2,3} \sum_{v_{i}, \bar{v}_{i}} \int \mathrm{d} E_{v} \frac{\mathrm{d} \phi_{v}}{\mathrm{~d} E_{v}} \omega_{v_{i}}\left(E_{v}\right) \int_{0}^{1} \mathrm{~d} y \mathcal{E}\left(y E_{v}\right) \frac{\mathrm{d} \sigma_{v_{i} N}}{\mathrm{~d} y} .
$$




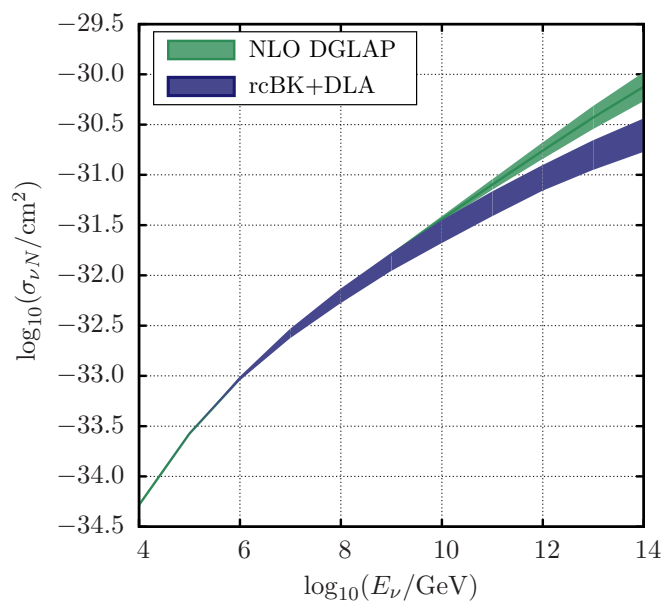

Figure 3. Neutrino-nucleon cross section calculated with NLO DGLAP with NNPDF3. 0 and with running coupling BK.

Table 1. Theoretical uncertainty in $\sigma_{v N}$ for different experiments covering the whole range of energies considered in this work.

\begin{tabular}{ccc}
\hline Experiment & Energy range $[\mathrm{GeV}]$ & $\sigma_{v N}^{\mathrm{DGLAP}} / \sigma_{v N}^{\mathrm{BK}}$ \\
\hline Auger [3] & $10^{8}-10^{10}$ & $\sim 1.3-1.7$ \\
ANITA-2 [21] & $10^{9}-10^{14}$ & $\sim 1.5-4.5$ \\
LUNASKA [22] & $\geq 10^{12}$ & $\gtrsim 2.5$ \\
\hline
\end{tabular}

It is proportional to the flux $\left(\phi_{v}\right)$, the exposure $(\mathcal{E})$ and the cross section $\left(\sigma_{v N}\right)$. Importantly, the exposures can also depend on the neutrino-nucleon cross section. The current upper bounds on the UHE neutrino flux have been obtained adopting the DGLAp $v$-nucleon cross sections. In table 1 we show how these bounds could be enlarged for different experiments due to the theoretical uncertainty coming from $\sigma_{v N}$. Neutrino radio-detection experiments based on the Askaryan effect, such as ANITA-2 and LUNASKA, explore the extremely high energy range. They are the best playground to test our predictions that start to differ significantly from those of DGLAP for $E_{v} \gtrsim 10^{8} \mathrm{GeV}$. We have shown that for the energies explored by the LUNASKA experiments, $E_{v} \sim 10^{14} \mathrm{GeV}$, the current upper bounds could be enlarged by a factor of up to $\sim 4.5$.

\section{Conclusions}

In summary, we have presented a quantitative study of the neutrino-nucleon total cross section in two different QCD approaches: NLO DGLAP and running coupling BK evolution. We have used state-of-the-art parametrizations of the parton distribution functions, in the DGLAP case, and of the dipole-nucleon scattering amplitude in the BK case. These parametrizations have been successfully tested against the available experimental data from HERA and the LHC. The cross sections obtained in the BK framework are systematically smaller than those calculated within the DGLAP framework, the difference between these two approaches bein as large as a factor 4.5 at the hightest neutrino energy studied in this work $E_{v}=10^{14} \mathrm{GeV}$. These systematic differences in the theoretical calculation of the total neutrino-nucleon cross section affect directly the experimental determination of the neutrino 
fluxes of astrophysical origin. Our current understanding of the hadronic structure would be improved by an electron-ion collider thus reducing the theoretical uncertainty of $\sigma_{v N}$.

\section{Acknowledgements}

This work has been supported by the Spanish MINECO (FPA2013-47836 and Consolider-Ingenio MultiDark CSD2009-00064) and by Junta de Andalucía (FQM101, 3048, 6552). The research of J.L.A. is also funded by a Ramón y Cajal contract of MINECO and by the QCDense Career Integration Grant of the FP7 Framework Program for Research of the European Commission, reference number CIG/631558.

\section{References}

[1] J.L. Albacete, J.I. Illana, A. Soto-Ontoso, Phys. Rev. D92, 014027 (2015), 1505.06583

[2] M. Aartsen et al. (IceCube), Phys.Rev.Lett. 111, 021103 (2013), 1304. 5356

[3] A. Aab et al. (Pierre Auger), Phys.Rev.D (2015), 1504.05397

[4] C. James, R. Ekers, J. Alvarez-Muniz, J. Bray, R. McFadden et al., Phys.Rev. D81, 042003 (2010), 0911. 3009

[5] C.L. Carilli, S. Rawlings, New Astron.Rev. 48, 979 (2004), astro-ph/0409274

[6] R.D. Ball et al. (NNPDF), JHEP 1504, 040 (2015), 1410. 8849

[7] A. Martin, W. Stirling, R. Thorne, G. Watt, Eur.Phys.J. C63, 189 (2009), 0901 . 0002

[8] I. Balitsky, G.A. Chirilli, Phys. Rev. D77, 014019 (2008), 0710.4330

[9] I.I. Balitsky, Phys. Rev. D 75, 014001 (2007), hep-ph/0609105

[10] Y. Kovchegov, H. Weigert, Nucl. Phys. A 784, 188 (2007), hep-ph/0609090

[11] J.L. Albacete, Y.V. Kovchegov, Phys. Rev. D75, 125021 (2007), arXiv: 0704 . 0612 [hep-ph]

[12] T. Lappi, H. Mäntysaari, Phys.Rev. D91, 074016 (2015), 1502.02400

[13] G. Beuf, Phys.Rev. D89, 074039 (2014), 1401.0313

[14] E. Iancu, J. Madrigal, A. Mueller, G. Soyez, D. Triantafyllopoulos, Phys.Lett. B744, 293 (2015), 1502.05642

[15] J.L. Albacete, N. Armesto, J.G. Milhano, C.A. Salgado, Phys. Rev. D80, 034031 (2009), 0902. 1112

[16] J.L. Albacete, N. Armesto, J.G. Milhano, P. Quiroga Arias, C.A. Salgado, Eur.Phys.J. C71, 1705 (2011), 1012. 4408

[17] J.L. Albacete (2015), 1507.07120

[18] K. Golec-Biernat, M. Wüsthoff, Phys. Rev. D59, 014017 (1998), hep-ph/9807513

[19] E. Iancu, K. Itakura, S. Munier, Phys. Lett. B590, 199 (2004), hep-ph/0310338

[20] G. Soyez, Phys. Lett. B655, 32 (2007), 0705. 3672

[21] P. Gorham et al. (ANITA), Phys.Rev. D82, 022004 (2010), 1003. 2961

[22] J. Bray, R. Ekers, P. Roberts, J. Reynolds, C. James et al., Phys.Rev. D91, 063002 (2015), 1502.03313 